\title{
Evolution of technique and results after permanent open repair for pectus deformities
}

\author{
Zachary W. Sollie, MD, Frank Gleason, MD, James M. Donahue, MD, and Benjamin Wei, MD
}

\section{ABSTRACT}

Objective: Open correction of pectus deformities has evolved since its origin. We performed a Ravitch type repair using a permanent titanium plate fixed with screws and describe the procedure with outcomes after our modifications.

Methods: A retrospective review of 61 pectus excavatum and pectus carinatum cases from August 2013 to April 2021 was performed. Data were extracted from medical records and reported. In January 2016, we began administering satisfaction surveys at the 6-month postoperative visit; results are reported.

Results: The mean age of our cohort was 24.5 years; $43(70 \%)$ were male. Fifty-four underwent pectus excavatum repair, 6 pectus carinatum repair, and 1 mixed repair. Median Haller index was 3.8. Mean operative duration was 98 minutes; mean blood loss was $116.4 \mathrm{~mL}$. Median chest tube duration was 5.0 days; median hospital stay was 4 days. Reexploration for bleeding was $30 \%$ in the first 10 patients. Protocol changes including postponing chemical deep vein thrombosis prophylaxis, using intraoperative hemostatic agents, and using shorter implantation screws decreased this to $० \%$ for the remaining cases. The most frequent complication was urinary retention $(21.3 \%)$. Postoperative surveys were completed for 37 of 50 patients. Seventy-five percent reported health improved, $65 \%$ reported exercise capacity improved, $75 \%$ reported breathing improved, and $59 \%$ reported chest pain improved. Self-esteem improved from $6.6 \pm 2.5$ (of 10) before surgery to $8.2 \pm 2.1$ after surgery. Ninety percent were satisfied and $86 \%$ would have the operation again.

Conclusions: Ravitch type repair with permanent titanium plate fixation is a safe and effective procedure for correction of pectus excavatum and carinatum. Most patients experience improvement in preoperative symptoms. (JTCVS Techniques 2022;12:212-9)

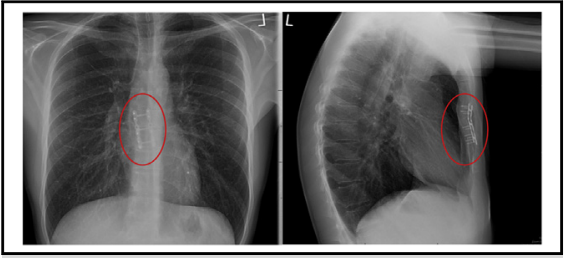

AP and lateral chest radiographs with visible titanium plate fixed with screws.

\section{CENTRAL MESSAGE}

Ravitch type repair using a permanent titanium plate is a safe, effective procedure for pectus deformities with high rates of satisfaction.

\section{PERSPECTIVE}

The Ravitch procedure for pectus deformities has evolved since its origin several decades ago. We perform a Ravitch type repair using a permanent titanium plate fixed with screws and have achieved good results. Our method increases efficiency of repair by eliminating the need for subsequent hardware removal while maintaining a high level of patient satisfaction.

See Commentary on page 220.
Video clip is available online.

The Ravitch type procedure for pectus excavatum (PE) and pectus carinatum (PC) was first reported by Mark Ravitch in 1949. ${ }^{1}$ It was originally described with extensive cartilage resection and suspension of the newly relocated sternum with stay sutures achieving good results. ${ }^{1,2}$ Modifications

From the Division of Cardiothoracic Surgery, UAB Department of Surgery, Birmingham, Ala.

Received for publication June 15, 2021; accepted for publication Dec 4, 2021; available ahead of print Jan 19, 2022.

Address for reprints: Benjamin Wei, MD, Division of Cardiothoracic Surgery, UAB Department of Surgery, Zeigler Research Building 701, 619 19th St South, Birmingham, AL 35233 (E-mail: bwei@uabmc.edu). have been made involving temporary internal stabilization devices to provide chest wall stability and prevent paradoxical respiratory motion. ${ }^{3-5}$ Although effective in providing sternal stability while healing, it has occasionally caused extensive pain or hardware complications such as migration. $^{4-9}$ Efforts have been made to improve singlestage operation by fixating the sternum with a permanent material that eliminates the need for hardware removal. ${ }^{7,8}$ Young and colleagues ${ }^{7}$ reported a technique using rib and sternal plating devices to provide permanent fixation and results of excellent satisfaction.

\section{6-2507}

Published by Elsevier Inc. on behalf of The American Association for Thoracic Surgery. This is an open access article under the CC BY-NC-ND license (http:// creativecommons.org/licenses/by-nc-nd/4.0/).

https://doi.org/10.1016/j.xjtc.2021.12.008 


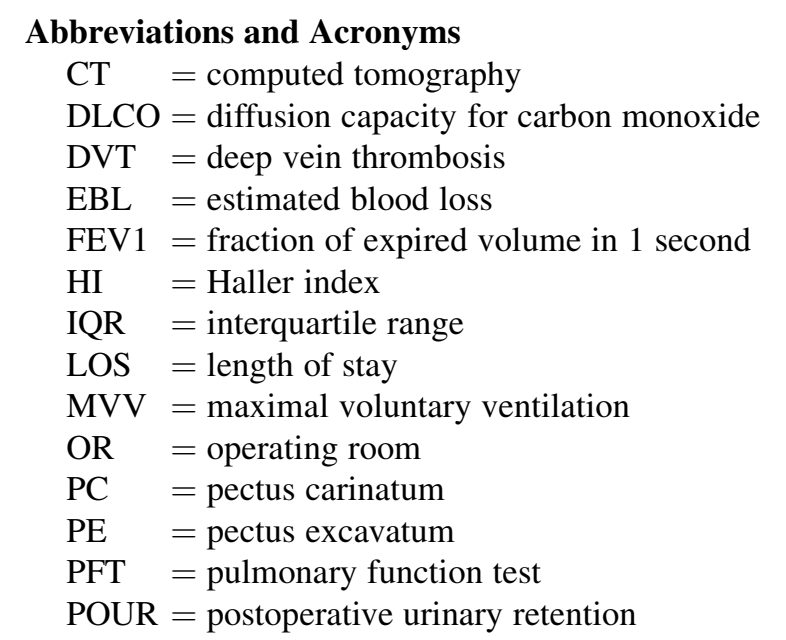

Multiple studies have compared open and minimally invasive techniques to understand the relative success associated with each method. ${ }^{3,6,10,11}$ Perioperative data show similar findings in operative times, estimated blood loss (EBL), and postoperative length of stay (LOS). ${ }^{6,10}$ Although the minimally invasive approach of the Nuss procedure offers several advantages, the Ravitch type repair has been shown to have a higher degree of long-term satisfaction. ${ }^{6}$ It is suggested that the highest satisfaction occurs when there is a complete correction without need for any reintervention. ${ }^{6}$ Furthermore, patients were more likely to choose the Ravitch procedure over the Nuss because of an aversion to having a metal bar left inside the chest. ${ }^{11}$ On the basis of these principles, a single-stage operation using a plate instead of a bar with long-term durability would be able to achieve good results with a high level of satisfaction.

At our institution, the Ravitch type repair is the primary method of correcting pectus deformities because of the expertise and experience of our surgeons. We have developed a technique using a permanent titanium plate fixed with screws that precludes the need for hardware removal, and that we surmise will prevent recurrence of the pectus deformity. The goals of our study are to report outcomes after our new developments with this procedure and offer insight about lessons learned through our experience. Video 1 outlines our findings.

\section{METHODS}

A retrospective review from internal medical records was conducted on 61 patients who received a Ravitch type repair for PE or PC deformities between August of 2013 and April of 2021. All data were extracted into a Microsoft excel file and analyzed for general statistical measures. The entire process was reviewed and approved for waiver of consent by our internal institutional review board (approval: IRB-300004194; January 6, 2020).
From initial patient evaluation, we gathered general cohort descriptors, preoperative pulmonary function test (PFT) results, and preoperative Haller index (HI). For PFTs, we used values representing the percent of predicted fraction of expired volume in 1 second (FEV1), maximal voluntary ventilation (MVV), and diffusion capacity for carbon monoxide (DLCO). Values were compared with postoperative PFTs at the 6-month clinic follow-up. HI was calculated from preoperative computed tomography (CT) scans by dividing the lateral measurements from the interior border of the rib cage by the distance between the anterior of the vertebral body and the posterior surface of the sternum at the most severe portion of the deformity. ${ }^{12}$ Data were collected for perioperative variables including operating room (OR) duration, EBL, number of days with a chest tube, LOS, and postoperative complications.

In January of 2016, we began administering a survey at 6 months post operation (Table 1). This survey is titled the single step questionnaire and has been used to assess quality of life outcomes in pectus operations. ${ }^{13}$ Results were analyzed for patient-centered outcomes.

\section{Procedure}

We perform a modified version of the Ravitch type repair for PE and PC deformities. Patients were brought into the OR and placed in the supine position. After sedation and intubation, they were prepped and draped in usual fashion leaving the anterior chest exposed. Paravertebral catheters were placed preoperatively, for postoperative administration of regional analgesia. For male patients, a midline vertical incision was made over the sternal deformity extending from the superior most margin of the deformity to the xiphoid process. For female patients, a bilateral submammary incision was made, with the central part of the incision curving cranially to help expose the area of maximal depression of the sternum. Bilateral pectoralis major flaps were created to achieve clear visualization of the sternum and parasternal costal cartilage. The location for the eventual sternal osteotomy was determined. An approximate $1-\mathrm{cm}$ piece of cartilage was resected in the location of maximum angulation of the deformed costochondral junction bilaterally below the level of the proposed osteotomy. This was performed with a 10 blade and a periosteal elevator protecting the tissue below each rib. Rongeurs were used to resect additional cartilage as deemed appropriate. The rectus abdominis muscle was transected approximately $2 \mathrm{~cm}$ below the insertion onto the lower sternum so that the fascia could be reapproximated at the end of the procedure. The posterior aspect of the sternum was dissected away from the mediastinum. A wedge osteotomy was performed through the sternum with an oscillating saw. The sternum was then repositioned (anteriorly for PE; posteriorly for PC) for favorable cosmetic and functional outcome. For most cases, we then fixed the sternum in place with a titanium SternaLock blue 12-hole ladder or 12-hole wide ladder plate (Zimmer Biomet). The plate was

Surgery Results
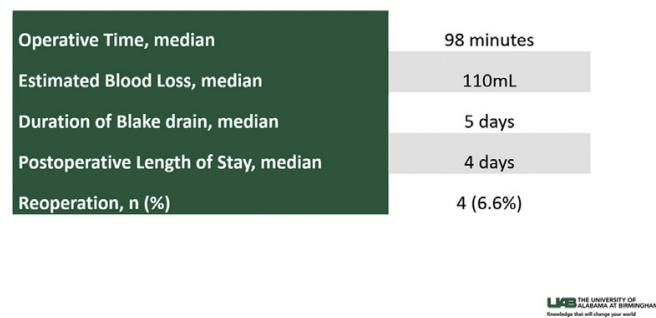

VIDEO 1. A video summarizing our intraoperative technique of permanent open repair of pectus deformities, as well as the findings in this study of 61 patients over 8 years. Video available at: https://www. jtcvs.org/article/S2666-2507(22)00019-0/fulltext. 
TABLE 1. Pectus excavatum/carinatum postoperative survey

\begin{tabular}{|c|c|c|c|c|}
\hline \multicolumn{5}{|c|}{ 1. How is your health in general after the operation? } \\
\hline Much better (5) & Somewhat better (4) & About the same (3) & Somewhat worse (2) & Much worse (1) \\
\hline \multicolumn{5}{|c|}{ 2. How is your exercise capacity now? } \\
\hline Much better (5) & Somewhat better (4) & About the same (3) & Somewhat worse (2) & Much worse (1) \\
\hline \multicolumn{5}{|c|}{ 3. How did the way your chest looked interfere with social activity BEFORE the operation? } \\
\hline Extremely (5) & Quite a bit (4) & Moderately (3) & Slightly (2) & Not at all (1) \\
\hline \multicolumn{5}{|c|}{ 4. How does the way your chest looks NOW interfere with social activity? } \\
\hline Not at all (5) & Slightly (4) & Moderately (3) & Quite a bit (2) & Extremely (1) \\
\hline \multicolumn{5}{|c|}{ 5. How satisfied are you with the overall postoperative appearance of your chest? } \\
\hline Extremely satisfied (5) & Very satisfied (4) & Satisfied (3) & Dissatisfied (2) & Very dissatisfied (1) \\
\hline \multicolumn{5}{|c|}{ 6. Are you bothered by the surgical scars? } \\
\hline Not at all (5) & Very slightly (4) & Slightly (3) & Somewhat (2) & $A \operatorname{lot}(1)$ \\
\hline \multicolumn{5}{|c|}{ 7. How has the operation affected your social life? } \\
\hline Major improvement (5) & Improved (4) & No change (3) & Worse (2) & A lot worse (1) \\
\hline \multicolumn{5}{|c|}{ 8. How has the operation affected your breathing? } \\
\hline Major improvement (5) & Improved (4) & No change (3) & Worse (2) & A lot worse (1) \\
\hline \multicolumn{5}{|c|}{ 9. How has the operation affected your level of chest pain? } \\
\hline Major improvement (5) & Improved (4) & No change (3) & Worse (2) & A lot worse (1) \\
\hline \multicolumn{5}{|c|}{ 10. How was your preoperative self-esteem? (Rate from 1-10: $1=$ poor, $10=$ excellent) } \\
\hline \multicolumn{5}{|c|}{ 11. How is your postoperative self-esteem? (Rate from 1-10: $1=$ poor, $10=$ excellent) } \\
\hline \multicolumn{5}{|c|}{ 12. How was your pain during your hospital stay? } \\
\hline None $(5)$ & Very mild (4) & Mild (3) & Moderate-severe (2) & Very severe (1) \\
\hline \multicolumn{5}{|c|}{ 13. How does pain interfere with your day-to-day activity now? } \\
\hline Not at all (5) & Very slightly (4) & Slightly (3) & Some (2) & A lot (1) \\
\hline \multicolumn{5}{|c|}{ 14. How is your pain now? } \\
\hline None (5) & Occasional (4) & Mild; no painkillers (3) & Moderate; painkillers (2) & A lot (1) \\
\hline \multicolumn{5}{|c|}{ 15. How do you feel about the final result? } \\
\hline Extremely satisfied (5) & Very satisfied (4) & Satisfied (3) & Dissatisfied (2) & Very dissatisfied (1) \\
\hline \multicolumn{5}{|c|}{ 16. How do you feel the chest looks now? } \\
\hline Major improvement (5) & Improved (4) & No change (3) & Worse (2) & Much worse (1) \\
\hline \multicolumn{5}{|c|}{ 17. Going back, would you have the operation again? } \\
\hline Yes & & & Unsure & No \\
\hline
\end{tabular}

bent anteriorly or posteriorly as appropriate, with the goal to place 4 screws cranially (above the osteotomy) and 6 screws caudally (below the osteotomy). Two screw holes overlie the osteotomy itself and were not used. We initially implanted the plate with $2.4-\mathrm{cm}$ diameter cancellous selfdrilling gold locking screws (Zimmer Biomet), which are designed to go through the anterior and posterior tables of the sternum. Later, we implanted the plate with the $2.7-\mathrm{cm}$ diameter cancellous magenta locking screws, to avoid having the tip of the screw protrude from the back of the sternum. A preoperative CT scan was used to determine sternum thickness at the location of hardware implantation, and to guide selection of the appropriate length screws. In deciding on length of each screw, we take into account the $0.8 \mathrm{~mm}$ thickness of the ladder plate and also whether or not the ladder plate is completely flush with the sternum at each individual screw hole. Hemostasis was achieved with a combination of standard electrocautery for specific bleeding, use of Aquamantys bipolar sealer (Medtronic) and Arista $\mathrm{AH}$ Absorbable hemostat $5 \mathrm{~g}$ (Becton Dickinson) for generalized bleeding, Surgicel (Johnson \& Johnson) for the gaps in the costal cartilage excised, and Gelfoam compressed sponge (Pfizer) for the osteotomy site. When adequate hemostasis was achieved, a 19-French Blake drain to bulb suction was placed in the space above the sternum, the pectoralis major advancement flaps reapproximated, and the skin closed and dressed appropriately. The patient was then extubated in the OR and sent to recovery.

Postoperative management focused on pain control and early mobilization. Modalities used include narcotic and local anesthetic delivered via paravertebral catheters, patient-controlled intravenous analgesia, oral narcotics pain medication, transdermal lidocaine patches, ibuprofen, and occasionally methocarbamol. We did not routinely place a Foley catheter in the OR, but would closely monitor patient for void checks in the immediate postoperative period. Intravenous furosemide was started on postoperative day 2 and given daily depending on drain output amount and serum level of blood urea nitrogen and creatinine, to decrease drain output before removal. Chemical deep vein thrombosis (DVT) prophylaxis was held until drain output was serous or thinly serosanguinous in nature, usually by postoperative day 3. Patients were discharged when pain was well controlled with oral medication, usually on postoperative days 4 or 5 . The drain 


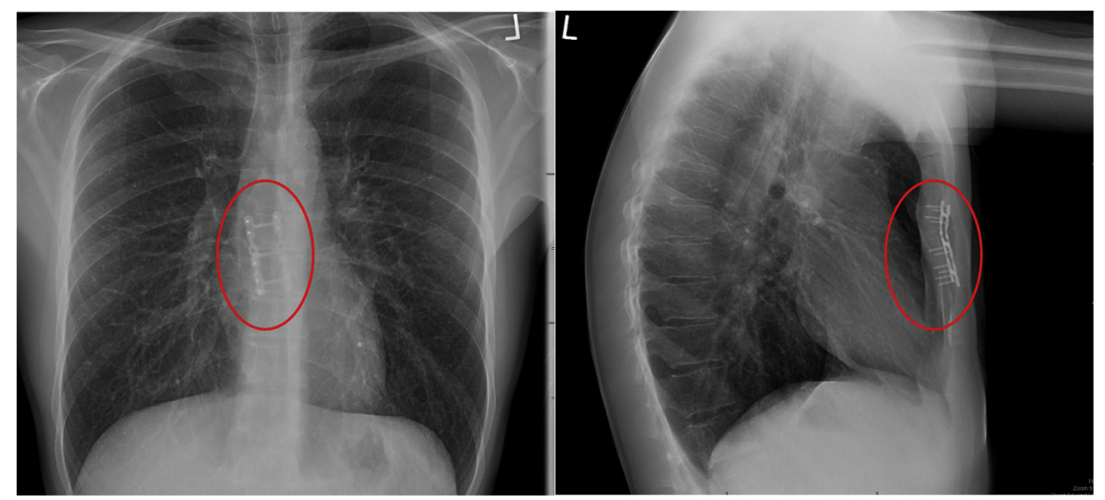

FIGURE 1. Anteroposterior and lateral chest radiographs with visible titanium plate fixed with screws.

was typically removed on the day of discharge, if daily output was $<100 \mathrm{~mL}$. Patients were seen for an early postoperative visit 4 to 6 weeks after discharge, and posterior-anterior and lateral chest radiographs were taken at that time (Figure 1). Patients then returned for a 6-month postoperative visit, at which time they received a patient survey and PFTs.

\section{RESULTS}

Sixty-one patients received Ravitch type repairs for PE or PC at our institution (Table 2). Fifty-four had a PE repair, 6 had a PC repair, and 1 had a mixed deformity repair. Median age was 20 (interquartile range [IQR], 19-26) years and 43 $(70 \%)$ were male. Median height and weight of the cohort was 180 (IQR, 168-185) $\mathrm{cm}$ and 65 (IQR, 57-75) $\mathrm{kg}$. Median preoperative HI was 3.8 (IQR, 3.2-4.5). Median operative duration was 98 (IQR, 90-107) minutes and EBL was 116.7 (IQR, 50-150) $\mathrm{mL}$. The median number of days with a chest tube was 5.0 (IQR, 4-5) days. Median LOS after the operation was 4 (IQR, 4-5) days. Preoperative PFTs had median values for FEV1 of $78 \%$ (IQR, $69 \%-89 \%$ ), MVV of $68 \%$ (IQR, $48 \%-82 \%$ ), and DLCO of $82 \%$ (IQR, $72 \%-96 \%$ ). Postoperative PFTs showed median values for FEV1 of 77\% (IQR, 68\%-85\%), MVV of $61.5 \%$ (IQR, $51 \%-73.8 \%$ ), and DLCO of $85 \%$ (IQR, $73 \%-94.5 \%$ ).

Reoperation occurred 4 (6.6\%) times over the course of our experience. Three were early for postoperative bleeding; the final was for hardware revision. In the first 2 cases, no obvious single source of bleeding was discovered but there was generalized oozing from the raw tissues involved in the dissection. The plate and repair were left intact in those cases. In the third case, a delayed injury to the right coronary artery causing cardiac tamponade had occurred. Reoperation was performed through the same incision by cutting the plate and retracting the sternum upward and laterally to expose the heart to ligate the injury. After hemostasis was achieved, the plate was reimplanted. The most common complication was postoperative urinary retention (POUR) in $13(21.3 \%)$ cases; followed by wound infection in $6(9.8 \%)$, postoperative bleeding in $3(5.0 \%)$, and pleural effusion in $3(5.0 \%)$. No patients experienced a lower extremity DVT or pulmonary embolism. One patient experienced a sigmoid sinus thrombus $(1.6 \%)$. The postoperative bleeding rate was $30 \%$ through the first 10 cases, which prompted a change in protocol involving delayed DVT prophylaxis and use of aggressive intraoperative hemostatic agents. Subsequent postoperative bleeding incidence was reduced to $0 \%$.

Thirty-seven postoperative surveys were collected of 50 (74\%) eligible patients (Table 3 ). Twenty-eight were male and the median age was 20 years. General health was reported to be better in 28 patients $(75 \%)$. Twenty-four $(65 \%)$ patients reported exercise capacity was better. Social activity was moderately to extremely affected by appearance before the operation in 19 patients $(51 \%)$ with only $8(22 \%)$ reporting the same after the operation. Thirty-three $(89 \%)$ reported satisfaction with appearance after the operation with $21(57 \%)$ of those reporting they were very or extremely satisfied. Only $3 \%$ reported being "somewhat" bothered by their surgical scars. The operation resulted in improved social life in $7(19 \%)$ and a major improvement in $4(11 \%)$. The operation led to improved breathing in 28 cases $(75 \%)$. Twenty-two $(59 \%)$ reported improvement in chest pain. Four (11\%) reported worsened chest pain. Mean self-esteem score improved from $6.6 \pm 2.5$ to $8.2 \pm 2.1$ of 10 from the pre- to postoperative state $(P<.05)$. Pain was moderate-severe during hospital stay in 20 cases $(54 \%)$ and very severe in 5 cases $(14 \%)$. At 6 months, 19\% reported "some" pain with day-to-day activity, with only $3 \%$ reporting needing "moderate" pain killers. Thirty-three patients $(90 \%)$ reported satisfaction with the overall outcome with $25(68 \%)$ of those reporting that they were very or extremely satisfied. Twenty-nine (79\%) reported improved appearance after the operation. If faced with the same decision, $32(86 \%)$ indicated that they would choose the operation again.

\section{DISCUSSION}

In the first 3 cases, we placed a Steinmann pin rather than the titanium plate. This pin was then removed at a later date, 
TABLE 2. Cohort data

\begin{tabular}{lc}
\multicolumn{1}{c}{ Characteristic } & Value \\
\hline Cases, $\mathrm{n}$ & 61 \\
Pectus excavatum & $54(88.5)$ \\
Pectus carinatum & $6(9.8)$ \\
Mixed deformity & $1(1.6)$ \\
\hline Age, years & $21(19-26)$ \\
Male sex & $43(70.5)$ \\
Height, cm & $180(168-185)$ \\
Weight, kg & $65(57-75)$ \\
Haller index & $3.8(3.2-4.5)$ \\
Surgery results & \\
OR duration (minutes) & $98(90-107)$ \\
EBL & $110(50-150)$ \\
Reoperation rate & $4(6.6)$ \\
Number of days with chest tube & $5(4-5)$ \\
Length of stay, days & $4(4-5)$ \\
Preoperative FEV1 & $78(69-89)$ \\
Preoperative MVV & $68(48-82)$ \\
Preoperative DLCO & $82(72-96)$ \\
Postoperative FEV1 & $77(68-85)$ \\
Postoperative MVV & $61.5(51-73.8)$ \\
Postoperative DLCO & $85(73-94.5)$ \\
Complications & \\
Urinary retention & $13(21.3)$ \\
Wound infection & $6(9.8)$ \\
Pleural effusion & $3(5.0)$ \\
Postoperative bleeding & $3(5.0)$ \\
Screw dislodgement with hardware revision & $1(1.6)$ \\
Coronary artery injury & $1(1.6)$ \\
Seroma & $1(1.6)$ \\
UTI & $1(1.6)$ \\
Sigmoid sinus thrombus & $1(1.6)$ \\
\hline Disce vas & \\
\hline
\end{tabular}

Discrete variables are listed as $\mathrm{n}(\%)$ and continuous variables are listed as median (interquartile range), except where otherwise noted. $O R$, Operating room; $E B L$, estimated blood loss; FEVI, forced expiratory volume in 1 second; $M V V$, maximal voluntary ventilation; $D L C O$, diffusion capacity for carbon monoxide; UTI, urinary tract infection.

a brief outpatient procedure using fluoroscopy to locate the head of the pin if it was not palpable beneath the skin. In 1 patient, persistent pain led to early removal of the pin. We also used the pin on procedure 56 in an attempt to avoid permanent hardware prosthesis impeding this patient's military career aspirations. Our protocol was changed after the first 3 cases, with use of a titanium plate fixed with screws to allow for sternal stabilization, which precluded the need for future hardware removal. A similar method has been reported and shows promising results. ${ }^{7}$ For our original method we used 16- to 18-mm self-drilling screws for fixation of the plate with the goal of locking the screws into the anterior and posterior cortical components of the sternum. However, these screws led to a serious complication in 1 case soon after adopting this technique involving injury to the branch of the acute marginal coronary artery from a screw protruding through the posterior sternum. The early postoperative course was unremarkable; however, several hours after the operation, he developed severe chest pain after being transferred from a stretcher to his bed, and soon thereafter went into shock. A transthoracic echocardiogram showed tamponade physiology suggesting hemorrhage into the pericardium. He required immediate reentry into the operating room, at which time the chest was reopened, the hemopericardium evacuated, and the coronary artery injury identified and ligated. The remaining recovery was uneventful. After this complication, hardware selection was immediately adapted to using "rescue" screws which lock into the anterior cortical structure of the sternum, and do not require fixation in the posterior table of the sternum for stability. After this adaption there were no further complications of this nature. Although there are no existing reports of this exact injury, Shaalan and colleagues $^{3}$ reported an instance of bleeding due to damage at the left internal mammary artery. Although these injuries are rare, their occurrence reiterates the importance of awareness of chest wall anatomy and demonstrates how even a small deviation from intended anatomical targets can lead to potentially catastrophic consequences.

Additionally, we instituted some protocol changes after 3 of our first 10 cases required reentry for bleeding. One of these reentries occurred before the patient left the OR and the remaining cases occurred within the first day (including the previously mentioned patient with the coronary injury). After our 10th case, we began aggressive use of intraoperative hemostatic agents and delayed initiation of chemical DVT prophylaxis until drain output became serous or thinly serosanguinous. After these adaptations there were no more bleeding complications. Bleeding is not an uncommon complication for this type of procedure and attention should be directed at mitigating its effects on overall outcomes. ${ }^{3,5}$

In our experience, the median operative time was 98 minutes. There are other reports of Ravitch type repairs with mean times ranging from 135 minutes to more than 250 minutes. ${ }^{3,6,7}$ Although operative time has a high degree of variability depending on the technique and skill of the surgeon, our results allude to a faster operation. The EBL within our study had a median of $110 \mathrm{~mL}$, which falls within the wide range of other reports of $33 \mathrm{~mL}$ to $359 \mathrm{~mL}$ with similar repairs. ${ }^{10,11}$ The median LOS among our cohort was 4 days, which is comparable with other reports. ${ }^{11}$ The shorter operative times in our study while maintaining acceptable EBL and LOS suggest that our method remains an efficient procedure.

There were no drastic improvements in PFTs in our study. This is consistent with previous studies that indicate minimal or decreased lung function, likely because of changes in chest wall compliance, after a Ravitch type 
TABLE 3. Survey results

\begin{tabular}{|c|c|}
\hline Variable & Value \\
\hline Number of responders & 37 \\
\hline Male sex & 28 \\
\hline Median age, years & $20(7.6)$ \\
\hline \multicolumn{2}{|c|}{ General health after operation } \\
\hline Much better & $16(43.0)$ \\
\hline Somewhat better & $12(32.0)$ \\
\hline About the same & $7(19.0)$ \\
\hline Much worse & $1(3.0)$ \\
\hline \multicolumn{2}{|c|}{ Exercise capacity after operation } \\
\hline Much better & $14(38.0)$ \\
\hline Somewhat better & $10(27.0)$ \\
\hline About the same & $7(19.0)$ \\
\hline Much worse & $3(8.0)$ \\
\hline \multicolumn{2}{|c|}{ Appearance interfered with social activity before operation } \\
\hline Not at all & $9(24.0)$ \\
\hline Slightly & $8(22.0)$ \\
\hline Moderately & $3(8.0)$ \\
\hline Quite a bit & $14(38.0)$ \\
\hline Extremely & $2(5.0)$ \\
\hline \multicolumn{2}{|c|}{ Appearance interfered with social activity after operation } \\
\hline Not at all & $17(46.0)$ \\
\hline Slightly & $11(30.0)$ \\
\hline Moderately & $5(14.0)$ \\
\hline Quite a bit & $2(5.0)$ \\
\hline Extremely & $1(3.0)$ \\
\hline \multicolumn{2}{|c|}{ Satisfaction with appearance after operation } \\
\hline Extremely satisfied & $13(35.0)$ \\
\hline Very satisfied & $8(22.0)$ \\
\hline Satisfied & $12(32.0)$ \\
\hline Dissatisfied & $2(5.0)$ \\
\hline Very dissatisfied & $1(3.0)$ \\
\hline \multicolumn{2}{|l|}{ Bothered by surgical scars } \\
\hline Not at all & $22(59.0)$ \\
\hline Very slightly & $8(22.0)$ \\
\hline Slightly & $5(14.0)$ \\
\hline Somewhat & $1(3.0)$ \\
\hline \multicolumn{2}{|c|}{ Operation effect on social life } \\
\hline Major improvement & $4(11.0)$ \\
\hline Improved & $7(19.0)$ \\
\hline No change & $22(59.0)$ \\
\hline Worse & $2(5.0)$ \\
\hline A lot worse & $1(3.0)$ \\
\hline \multicolumn{2}{|c|}{ Operation effect on breathing } \\
\hline Major improvement & $9(24.0)$ \\
\hline Improved & $19(51.0)$ \\
\hline No change & $6(16.0)$ \\
\hline \multicolumn{2}{|c|}{ Operation effect on level of chest pain } \\
\hline Major improvement & $8(21.0)$ \\
\hline Improved & $14(38.0)$ \\
\hline No change & $10(27.0)$ \\
\hline Worse & $4(11.0)$ \\
\hline
\end{tabular}

TABLE 3. Continued

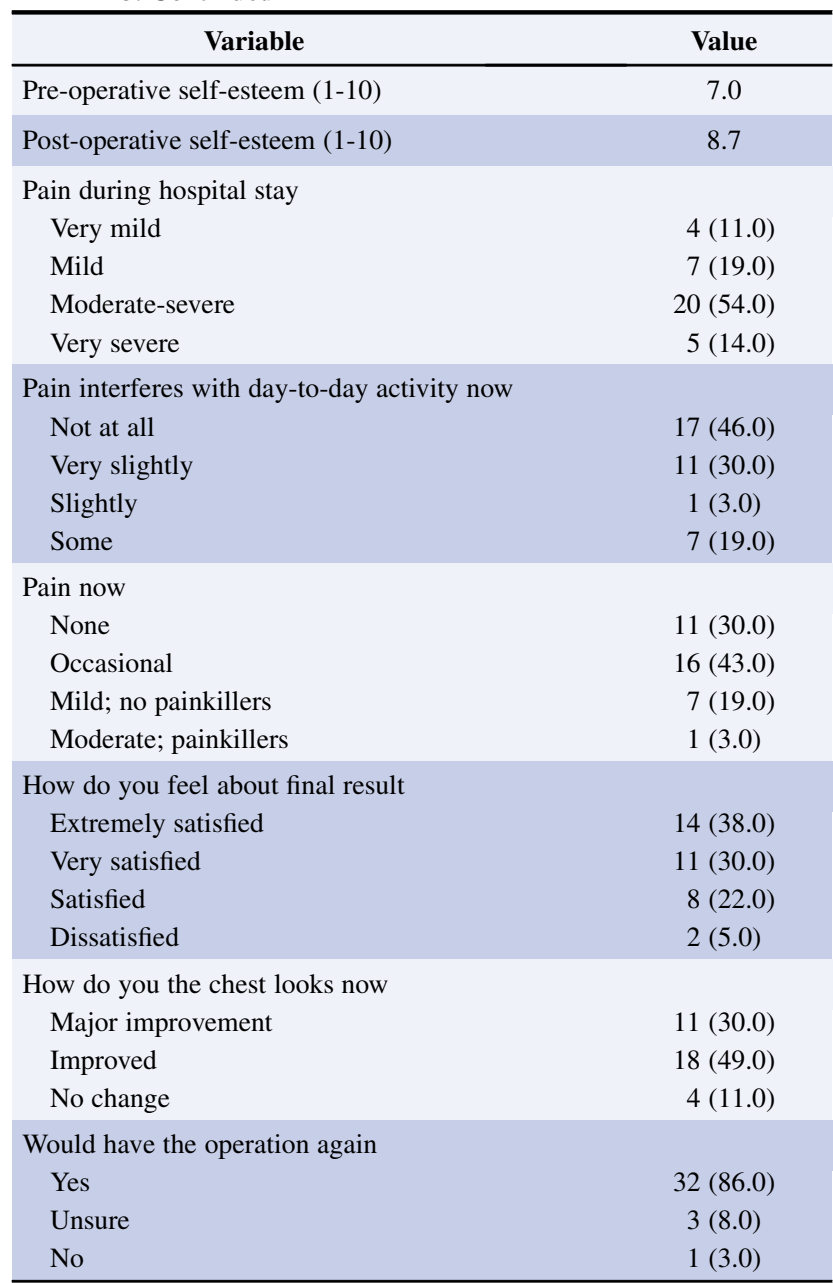

repair. ${ }^{14-16}$ Toward the end of our experience, the requirement for COVID testing the day before obtaining PFTs caused omission of postoperative studies in several patients, which limited our data set. However, $75 \%$ of patients reported a subjective improvement in breathing ability. Improvement in cardiopulmonary status likely relates more to mitigation of right ventricular and right atrial compression, and possibly restoration of normal tricuspid annular anatomy, than correction of respiratory mechanics. ${ }^{17}$ Among PC cases, $100 \%$ reported breathing improvement. This is consistent with previous reports of improvement in dyspnea after PC repair. ${ }^{18}$ The physiologic mechanism behind this finding is more elusive and is not well studied; however, we speculate that it might be related to improving the compliance of the chest wall and/or tidal volume. The improved exercise capacity reported in $65 \%$ of participants is quite possibly inter-related to the previously mentioned cardiorespiratory status. Further exploration could be beneficial to more clearly define these improvements. 
Although our method is effective at lifting the lower half of the sternum and providing additional space for the heart, the upper part of the chest above the osteotomy is not modified and often remains somewhat "sunken." Additionally, the areas where the costal cartilages jut out laterally might still be obvious, because the cartilage resected does not change the contour of the costochondral junction. If these areas remain "high" after the plate is implanted, a rongeur can be used to "lower" the maximum anterior protrusion of these cartilages, but we try to avoid resecting too much to prevent an excess gap in the chest wall. Second, we recommend that patients be interviewed about expectations for repair, to determine the relative magnitude of cosmetic versus cardiopulmonary concerns. In the uncommon situation that cosmetic concerns seem paramount or if the patient is focused on the size and nature of the incisions, we might recommend they seek an opinion on adult Nuss repair. Third, we aim for an intraoperative "overcorrection" of an excavatum deformity because when the rectus is reapproximated, the skin is closed, and later, when the postoperative swelling and/or seroma has resolved, any protrusion of sternum appreciated intraoperatively becomes much less noticeable. Finally, although recurrence of the deformity with hardware appearance is rare, we have seen it in a single case involving a male adolescent. In the year after the operation, he grew significantly. We speculate that continued evolution and pliability of his chest wall led to recurrence. Therefore, we try to wait until patients are done or nearly done growing before offering this repair. Despite these previously mentioned limitations, we can still conclude from our surveys that our patient population was satisfied with their repairs.

The 2 most frequent complications were POUR (21.3\%) and wound infection $(9.8 \%)$. POUR is a known and often anticipated complication across many surgical specialties. Specifically, in major thoracic operations, efforts have been made to predict this complication to determine the need for prophylactic intervention. ${ }^{19}$ Wound infection is always a risk with surgery, and measures should be taken to minimize the incidence. We found the rate of wound infection was somewhat high. This is likely because of a combination of factors, including traction on the skin to get exposure to the costal cartilages to be resected and the extensive length of incision at risk when performing the bilateral submammary incision. We have started using negative-pressure closed incision dressings, which have been shown to improve skin perfusion, increase wound strength, and minimize drainage in animal models. ${ }^{20}$ Wound infections (any wound dehiscences, which were all limited to a small 1- to $3-\mathrm{cm}$ portion of the incision, were counted as and considered infections) were treated with oral antibiotics. No patients developed infection of the sternal hardware or required operative reexploration for infection. The final complication to mention was unexpected but significant. On case 51, the patient experienced extraordinary coagulation complications including thrombosis in her sigmoid sinus and internal jugular vein extending to the subclavian. The etiology was unclear, because the manubrium was not manipulated during the operation. However, a retrospective review of the preoperative CT scan showed fairly significant compression of the innominate vein. Perhaps the combination of postoperative hypercoagulability and a subtle decrease in the anteroposterior dimension of the upper chest cavity because of the carinatum repair was enough to result in thrombus of this vessel and those upstream. Aside from this, she achieved significant improvement of her chest symptoms after surgery. However, the postoperative morbidity caused by this complication deterred her from choosing to have the operation again.

Although short-term satisfaction is favorable, the 6-month follow up time frame of our study offers some limitations. This time frame is simply too short to understand the full extent of long-term satisfaction with these operations. In an era of increasing patient access via telemedicine, it might be feasible to increase follow-up time with this same cohort over a period of years. Another obvious limitation is the absence of repeat CT scans of the chest to calculate postoperative HI. However, we obtained lateral view chest radiographs to confirm appropriate angulation of the sternum below the osteotomy as well as verify hardware positioning, integrity, and fixation into the sternum. Although the chest radiographs provided the necessary clinical information in our cases, future studies might consider a repeat CT scan for having a consistent comparison for pre- and postoperative HI. However, researchers must account for the increased costs and radiation exposure associated with this added feature. Three-dimensional surface imaging might also be a promising technique to document changes in the $\mathrm{HI}$ without exposing patients to additional and unnecessary ionizing radiation. Considering the improvements in symptomatology shown in the surveys, we believe that this version of the modified Ravitch repair is an effective method of correcting PE and PC.

Finally, some surgeons might argue that a Nuss procedure offers advantages over the Ravitch procedure. We believe that although the Nuss procedure is likely more cosmetically appealing and offers a decreased wound complication rate and less blood loss compared with the Ravitch, the 2 techniques are similar in terms of recovery and LOS, and the Nuss operation might actually be more painful. ${ }^{21}$ In addition, the Nuss procedure can be more difficult in adult patients who have a more ossified chest wall; some patients can require a hybrid approach requiring open osteotomy and chondroplasty, which could presumably negate some of the advantages of the Nuss operation. $^{22}$ Furthermore, pectus repair via the Nuss 
procedure necessitates a second operation to remove the bar, which some patients deem undesirable. We believe that both operations can offer excellent results; large studies have shown similar rates of recurrence and postoperative complications for the 2 operations in the adult population. ${ }^{23}$

\section{CONCLUSIONS}

The protocol we adopted for pectus repairs at our institution sufficiently corrects the deformity while presumably eliminating the need for a further operation to remove hardware. Although rare serious complications can occur, short-term results are favorable and patients are satisfied on the basis of postoperative survey results. Although this protocol needs further studies to determine long-term outcomes, the preliminary results are promising for continued success.

\section{Conflict of Interest Statement}

The authors reported no conflicts of interest.

The Journal policy requires editors and reviewers to disclose conflicts of interest and to decline handling or reviewing manuscripts for which they may have a conflict of interest. The editors and reviewers of this article have no conflicts of interest.

The authors thank all of the supporting staff within our division who have provided us with the resources necessary to conduct our investigations.

\section{References}

1. Ravitch MM. The operative treatment of pectus excavatum. Ann Surg. 1949;129: 429-44.

2. Mansour KA, Thourani VH, Odessey EA, Durham MM, Miller JI Jr, Miller DL. Thirty-year experience with repair of pectus deformities in adults. Ann Thorac Surg. 2003;76:391-5.

3. Shaalan AM, Kasb I, Elwakeel EE, Elkamali YA. Outcome of surgical repair of Pectus Excavatum in adults. J Cardiothorac Surg. 2017;12:72.

4. Fonkalsrud EW, DeUgarte D, Choi E. Repair of pectus excavatum and carinatum deformities in 116 adults. Ann Surg. 2002;226:304-12.

5. Tikka T, Kalkat MS, Bishay E, Steyn RS, Rejesh PB, Naidu B. A 20-year review of pectus surgery: an analysis of factors predictive of recurrence and outcomes. Interact Cardiovasc Thorac Surg. 2016;23:908-13.

6. Kang CH, Park S, Park IK, Kim YT, Kim JH. Long-term surveillance comparing satisfaction between the early experience of Nuss procedure vs. Ravitch procedure. Korean J Thorac Cardiovasc Surg. 2012;45:308-15.
7. Young S, Lau ST, Shaul DB, Vazquez WD, Yoo ET, Sydorak RM. A new technique in complex chest wall reconstruction: open reduction and internal fixation. J Pediatr Surg. 2018;53:2488-90.

8. Karagounis VA, Wasnick J, Gold JP. An innovative single-stage repair of severe asymmetric pectus excavatum defects using substernal mesh bands. Ann Thorac Surg. 2004;78:19-21.

9. Onursal E, Toker A, Bostanci K, Alpagut U, Tireli E. A complication of pectus excavatum operation: endomyocardial steel strut. Ann Thorac Surg. 1999;68: 1082-3.

10. Mao YZ, Tang S, Li S. Comparison of the Nuss versus Ravitch procedure for pectus excavatum repair: an updated meta-analysis. J Pediatr Surg. 2017;52: $1545-52$.

11. Elsayed HH, Hassaballa AS, Abdel Hady SM, Elsbastawisy SE, Ahmed TA Choosing between the modified Ravitch and Nuss procedures for pectus excavatum: considering the patients's perspective. Ann R Coll Surg Engl. 2016;98: 581-5.

12. Haller J, Alex J, Kramer SS, Lietman SA. Use of CT scans in selection of patients for pectus excavatum surgery: a preliminary report. J Pediatr Surg. 1987;22: 904-6.

13. Krasopoulos G, Dusmet M, Ladas G, Goldstraw P. Nuss procedure improves the quality of life in young male adults with pectus excavatum deformity. Eur J Cardiothorac Surg. 2006;29:1-5.

14. Jayaramakrishnan K, Wotton R, Bradley A, Nadu B. Does repair of pectus excavatum improve cardiopulmonary function? Interact Cardiovasc Thorac Surg. 2013;16:865-70.

15. Jaroszewski DE, Ewais MM, Chao C, Gotway MB, Lackey JJ, Myers KM, et al. Success of minimally invasive pectus excavatum procedures (modified Nuss) in adult patients ( $>$ /=30 years). Ann Thorac Surg. 2016;102:993-1003.

16. Johnson JN, Hartman TK, Pianosi PT, Driscoll DJ. Cardiorespiratory function af ter operation for pectus excavatum. J Pediatr. 2008;153:359-64.

17. Salama AY, Arisha MJ, Nanda NC, Klas B, Ibeche B, Wei B. Incremental value of three-dimensional transthoracic echocardiography over the two-dimensional modality in the assessment of right heart compression and dysfunction produced by pectus excavatum. Echocardiography. 2019;36:150-63.

18. Fonkalsrud EW. Surgical correction of pectus carinatum: lessons learned from 260 patients. J Pediatr Surg. 2008;43:1235-43.

19. Kim KW, Lee J, Kim JS, Lee Y, Choi W, Jung H. Risk factors for urinary retention following minor thoracic surgery. Interact Cardiovasc Thorac Surg. 2015; 20:486-92.

20. Suh H, Lee A, Park EJ, Hong JP. Negative pressure wound therapy on closed surgical wounds with dead space: animal study using a swine model. Ann Plast Surg. 2016;76:717-22.

21. Papic JC, Finnell SM, Howenstein AM, Breckler F, Leys CM. Postoperative opioid analgesic use after Nuss versus Ravitch pectus excavatum repair. J Pediatr Surg. 2014;49:919-23.

22. Velazco CS, Arsanjani R, Jaroszewski DE. Nuss procedure in the adult population for correction of pectus excavatum. Semin Pediatr Surg. 2018;27:161-9.

23. Toci GR, Davis TA, Bigelow BF, Yang SC. Analyzing outcomes of Nuss and Ravitch repair for primary and recurrent pectus excavatum in adults. Ann Thorac Surg. 2020;110:272-5.

Key Words: pectus excavatum, pectus carinatum, Ravitch type repair, permanent, titanium plate 